\title{
FIELD DEPLOYMENT OF AN AMBIENT VIBRATION-BASED SCOUR MONITORING SYSTEM AT BAILDON BRIDGE, UK
}

\author{
KKGKD Kariyawasam ${ }^{*}$, PRA Fidler, JP Talbot and CR Middleton \\ Department of Engineering, University of Cambridge, United Kingdom \\ * Corresponding author
}

\begin{abstract}
Scour, the loss of material around bridge foundations due to hydraulic action, is the main cause of bridge failures in the United Kingdom and in many other parts of the world. Various techniques have been used to monitor bridge scour, ranging from scuba divers using crude depth measuring instrumentation to high-tech sonar and radar-based systems. In contrast to most other techniques, vibration-based scour monitoring uses accelerometers to provide real-time monitoring whilst also being robust and relatively simple to install. This is an indirect technique that aims to measure changes in the dynamic response of the structure due to the effects of scour, rather than attempting to measure scour directly. To date, research on vibration-based scour monitoring has been limited to laboratory-based experiments and numerical simulations, both of which have indicated that the natural frequencies of bridges should indeed be sensitive to scour. Due to pre-existing scouring, and planned repair work, Baildon Bridge in Shipley, Yorkshire provided a rare opportunity to validate vibration-based scour monitoring in both a scoured and a repaired state. A sensor system was deployed with 10 Epson low-noise, high-sensitivity accelerometers to measure the ambient vibration of the bridge before, during, and after the repair. This paper describes the installation of the accelerometer-based system, the numerical modelling of the bridge and the model updating carried out with the initial findings. Initial operational modal analysis has found two consistent vibration modes of the bridge that were scour sensitive according to the updated numerical model. But the variability of the measured frequencies, compared to the expected scour induced change in frequency, indicates a potential challenge for monitoring scour of small span bridges with vibration-based methods.
\end{abstract}

\section{Introduction}

Scour and flooding reportedly accounts for up to $50-60 \%$ of historical bridge failures (Shirole and Holt, 1991; Wardhana and Hadipriono, 2003) and studies have also shown that scour is the leading cause of bridge failure around the world [see studies from New Zealand (Melville and Coleman, 2000), Taiwan (Ko et al., 2010), USA (Hunt, 2009) and UK (Dikanski et al., 2016). Bridge scour monitoring techniques range from simple sounding rod devices to autonomous underwater vehicles (CIRIA, 2015; Murphy et al., 2011). However, most of these techniques have numerous drawbacks related to continuity, reliability and durability under flood loading.

Most of the limitations with the current techniques could potentially be avoided by an indirect technique, vibrationbased scour monitoring, which correlates the change in support condition due to increased scour depth with a resulting shift of certain natural frequencies of the bridge (Prendergast et al., 2013). This technique has been widely adopted on railway bridges in Japan, where the impact of iron balls is used as a source of impulsive excitation (Masui and Suzuki, 2009; Shinoda et al., 2008). However, to the authors knowledge, this technique has not been widely adopted in other countries, perhaps in part due to the practicalities of the excitation method. With the recent advances in high-sensitivity accelerometers (Zhu et al., 2018), it is possible to obtain the natural frequencies of bridges using low-level ambient vibration due to sources such as traffic or wind (Brownjohn et al., 2017; Rehman et al., 2016).
To date, the use of natural frequencies to monitor bridge behaviour has been centred around detecting physical damage to the structure itself (Seo et al., 2016). However, this is not a sensitive method; for example, physical damage, such as bridge deck cracks, has been found to not cause a significant change of natural frequency (Chang and Kim, 2016). In contrast, with scour, the natural frequency has been found to vary by double-digit percentages (Prendergast et al., 2016).

While the sensitivity of the natural frequency to scour has been demonstrated with numerical models, there have only been very limited field studies on the topic. Research monitoring of scour on real bridges is difficult as scour is hard to predict - a monitoring system would need to be installed on a candidate bridge with no guarantee that natural scouring would occur within the typical timespan of a research project. However, at Baildon Bridge, due to the pre-existing scouring and the planned repair work, a rare opportunity arose to monitor the bridge in both a scoured state, a repaired state, and at various intermediate states as the repairs progressed. As the repairs involved a significant, but controlled, variation of the level of the river bed, this was also an opportunity to validate vibrationbased techniques such as modal analysis for use in scour monitoring. The repair works allow for monitoring of the bridge as the river bed is raised - in effect, scour in reverse. This paper describes the numerical model development and scour simulation, sensor system development, initial experimental results and the updating of the finite-element model. 


\section{Baildon Bridge}

Baildon Bridge in Shipley UK was reconstructed in 1931 as a beam slab type three-span bridge. It carries the four-lane Otley Road (A6038) over the river Aire. As shown in Figure 1, Baildon Bridge has two concrete piers constructed on pile foundations in the river. The bridge is $20 \mathrm{~m}$ wide, and $23 \mathrm{~m}$ in total length. Each pier is $4.55 \mathrm{~m}$ tall, $0.75 \mathrm{~m}$ thick and $21.5 \mathrm{~m}$ wide.

\section{Figure 1 Elevation of Baildon Bridge}

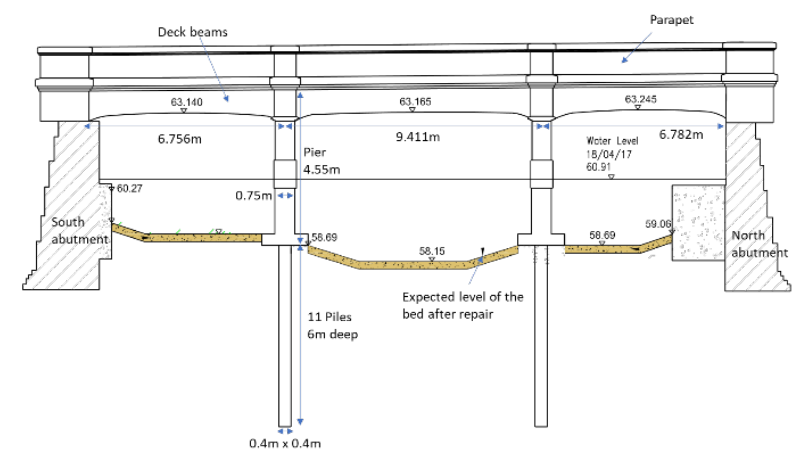

After a flooding event in 2015, the bridge was inspected using divers and the south pier of the bridge was found to have $1.8 \mathrm{~m}$ of scouring on one side. Bradford Metropolitan Council planned a repair programme to refill this scour hole with up to $0.6 \mathrm{~m}$ of soil topped by a concrete bag wall and concrete mat (Figure 2).

Figure 2 Scour refill proposed at the south bridge pier bed level before repair

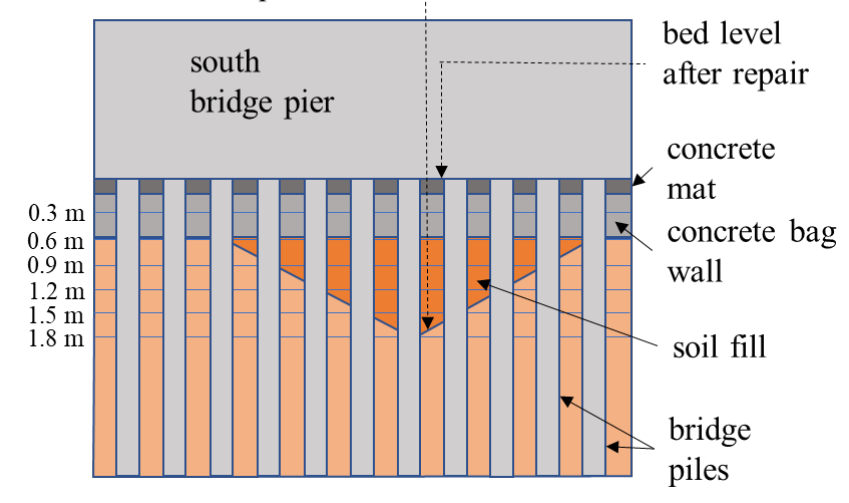

\section{River bed scanning with Sonar}

The river bed profile was scanned with sonar using the BridgeCat mobile bridge inspection system before the scour repair works started. The BridgeCat system consists of a Unimog all-terrain vehicle fitted with a crane capable of extending $14.8 \mathrm{~m}$ below the level of the crane base (Roberts, 2017). With the vehicle parked on the pedestrian pavement near the parapets, the crane was able to reach all locations of interest under the bridge. Once the crane was extended to a desired location underwater, a sonar scanning head at the end of the crane captured 2D sonar images. See Figure 3. BridgeCat scanning of the full bridge was carried out over 6 hours during off-peak daytime traffic after 09:00.

Figure 3: BridgeCat vehicle taking sonar scans before repair

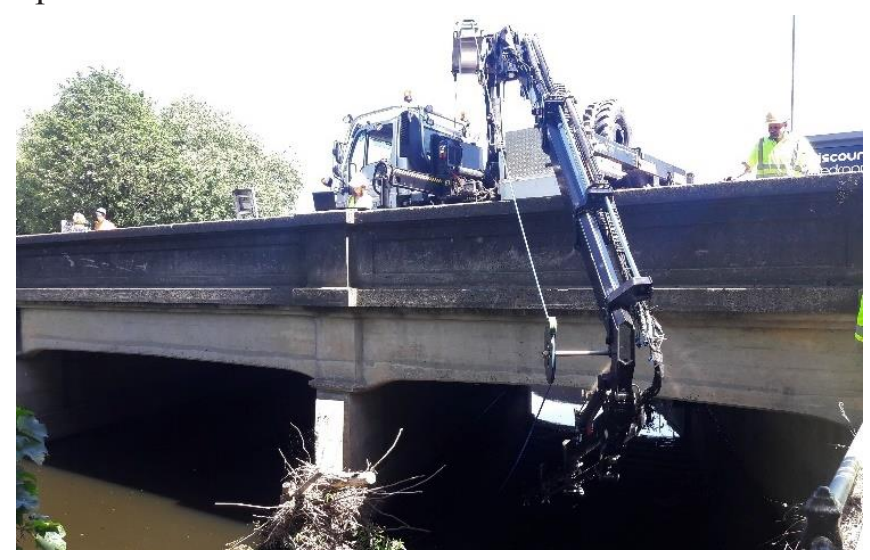

The scour depth profile produced by the sonar imagery showed good agreement with the profile shown in diving inspection reports (Figure 4).

Figure 4: Elevation of the maximum scour levels as shown in the dive inspection report and in sonar imagery.

(Sonar images provided by Jenny Roberts, Gaist Solutions)

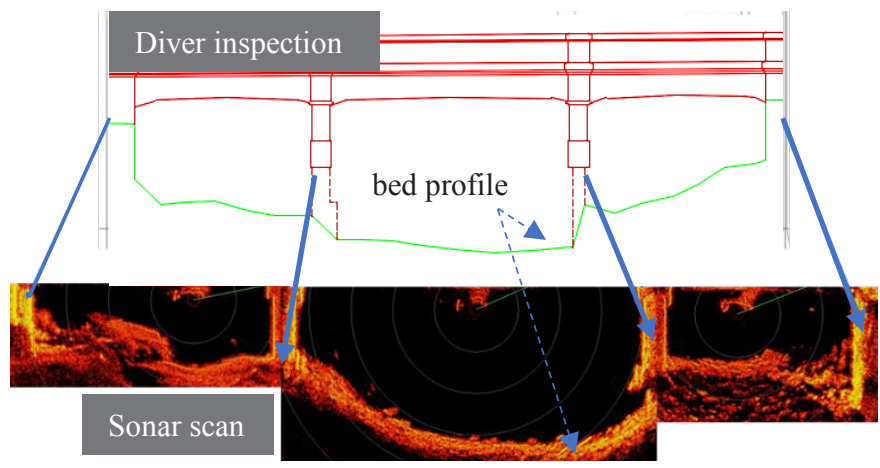

\section{Initial scour simulation}

\subsection{Finite-element model}

A finite-element model was created with the CSiBridge2017 structural analysis software, to model the dynamic behaviour of Baildon Bridge and to simulate the effect of scour. Figure 5 shows the finite elements used to represent the bridge.

The bridge deck slab was represented by shell elements that model both membrane and plate bending behaviour. Bridge deck beams and piles were represented by frame elements, which use a three-dimensional beam/column formulation. The soil-pile interaction was represented by a Winkler spring model. Winkler model represents the soil medium as a system of discreet, mutually independent and linear elastic springs (Dutta and Roy, 2002). 
Figure 5 Finite-element model developed to represent Baildon Bridge

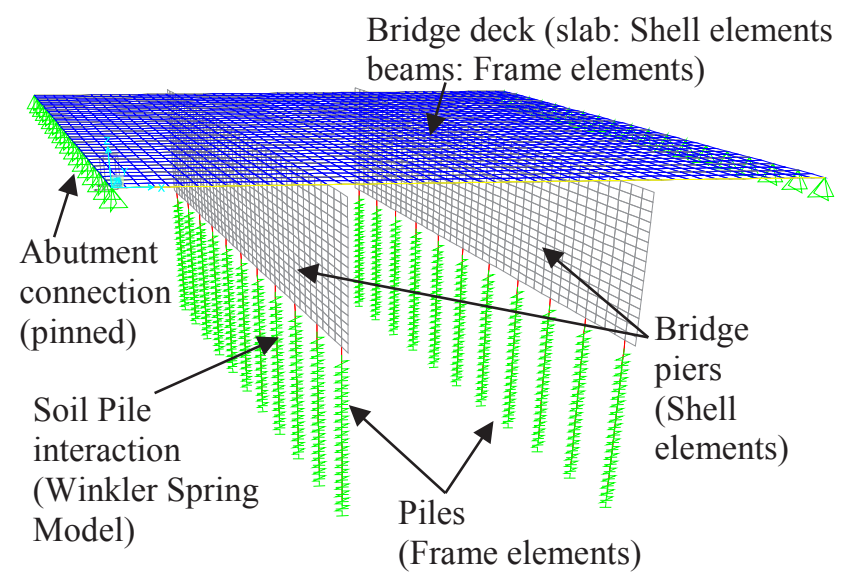

Some of the properties of the bridge were not available at the time of modelling and assumptions were therefore made based on engineering judgement. The concrete grade of the piles, pier and deck beams was assumed to be the same as that indicated for the deck slab (C40). Underwater inspection and two borehole records from 1930 identified the soil type up to the pile driven depth as sandy gravel. The soil was therefore assumed as medium dense sand with $50 \%$ relative density.

The bridge deck slab has two sections: an original $150 \mathrm{~mm}$ thick slab; and a $65 \mathrm{~mm}$ top layer that was added in 1995. This top layer is dowelled into the existing slab using threaded studs and hence a 'no slip' connection could be assumed. The deck is therefore considered as a $215 \mathrm{~mm}$ composite concrete slab.

The piers are located with a skew, and hence the size of the deck elements in the longitudinal direction was chosen such that the pier could be connected to the deck while keeping the accurate skew.

The beams were offset from the slab to provide the composite beam slab behaviour. The slab sections were placed in between these beam sections. The reinforcement arrangement indicates a continuous connection of bridge beams over the piers. The deck beams are coupled with diaphragm beams which rest on bridge piers. Diaphragm beams also connect the deck beams at the bridge abutments in a similar manner. The concrete diaphragm beam and pier/abutment top interface has significant friction; hence the connection can be assumed to provide full translational stiffness. Although there is no reinforcement connecting the piers to the deck, some bending stiffness would be present at this interface due to friction. Therefore, an integral (fully fixed) connection was assumed at beam-pier interface under low-intensity ambient vibration caused by traffic.
Table 1 Mode shapes captured with the initial FE model and the expected frequency shifts due to scour

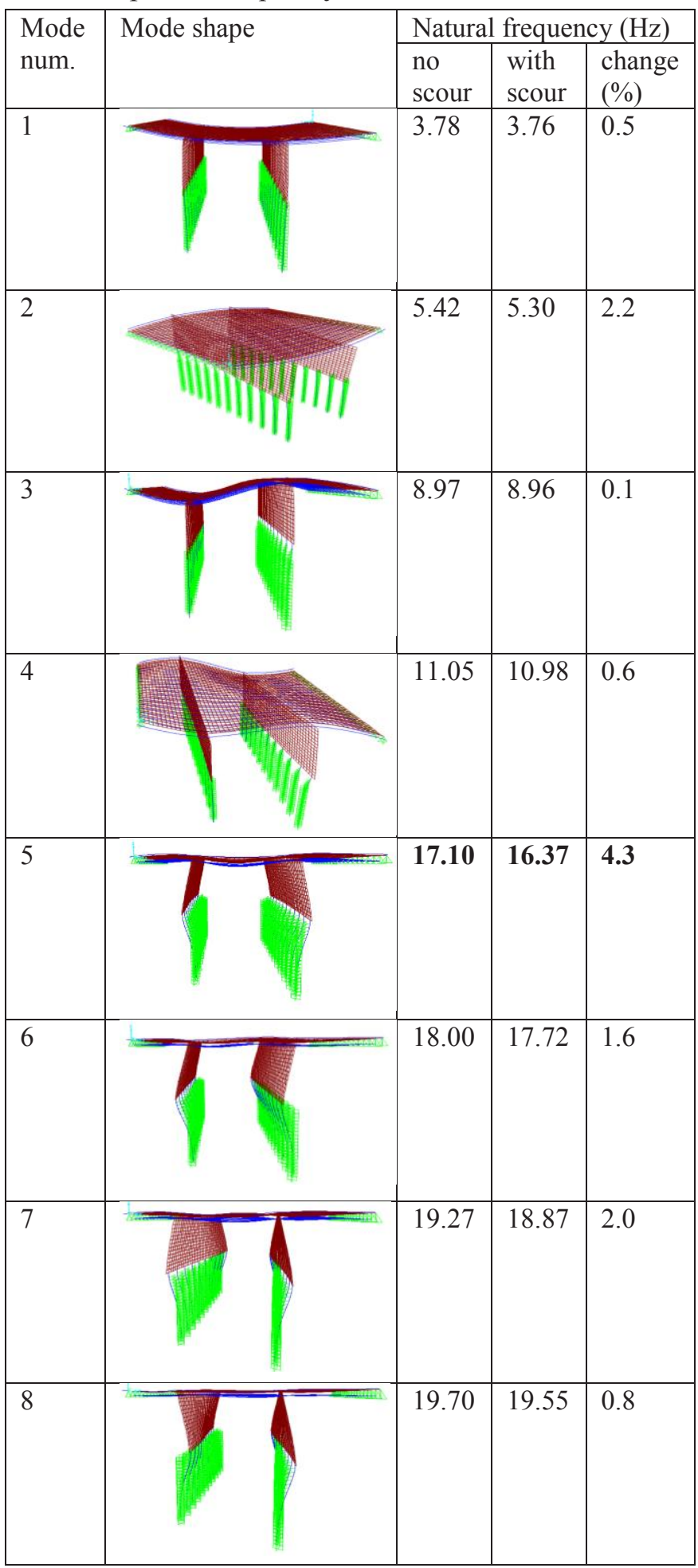

Winkler model, although approximate, provides a means by which the soil restraint at the pile heads may be varied to model varying degrees of scour. The Winkler spring stiffness of the soil was calculated using the API design code (API, 2002). As explained in Prendergast et al. (2017), the horizontal spring stiffnesses were calculated assuming small-strain behaviour. 
To provide 3D behaviour, small-strain spring stiffnesses were provided down the piles in both the transverse and longitudinal directions. In the vertical direction, the spring stiffness was taken as the tangent stiffness based on the large-strain value under the dead load of the bridge, as explained in CSI (2017).

A standard, linear modal analysis was conducted to determine the undamped vibration modes of the system.

\subsection{Initial scour simulation}

The refurbished bridge after filling of the scour hole was first modelled with soil springs as explained in Section 4.1. The effect of the scour was then simulated by removing the springs at the locations where the scour hole was present. The effect of the concrete bag wall and concrete mat was ignored in the initial analysis.

Table 1 shows the initial analysis results for all vibration modes up to $20 \mathrm{~Hz}$. The first 4 modes are predominantly vertical. The first longitudinal mode, Mode 5, is the most scour sensitive, showing a $0.73 \mathrm{~Hz}(4.3 \%)$ reduction in natural frequency due to scour. It was therefore expected that scour refilling would show the reverse $(0.73 \mathrm{~Hz}$ increase in natural frequency). Instrumentation was therefore focused on capturing this mode of vibration.

\section{Instrumentation}

The initial modelling suggested that the natural frequencies of the bridge are relatively high, thus the response amplitude due to traffic was likely to be low. High-sensitivity and low-noise accelerometers were therefore needed. The accelerometers used for the deployment were Epson M-A550 QMEMS devices, which claim $0.5 \mu \mathrm{g} / \sqrt{ } \mathrm{Hz}$ output noise for the $0.5-6$ $\mathrm{Hz}$ frequency band, and $60 \mu \mathrm{g} / \sqrt{ } \mathrm{Hz}$ output noise for the $6-100$ $\mathrm{Hz}$ frequency band (EPSON, 2018). The M-A550 is supplied in two variants: one using RS-422 for digital communication and the other using CANBus. There is no analogue variant. As the intent of the monitoring was to record time-synchronised data from multiple sensors, the team elected to use the RS-422 variant, with each sensor connected to the data logger via a separate cable. Whilst the CANBus variant would have allowed for simpler cabling, it would only have allowed for collection of data from one sensor at a time in sequence, and so would not have been truly time-synchronised. An internal filter that uses a Keiser window with 512 tap and cut-off frequency of $20 \mathrm{~Hz}$ was chosen to avoid aliasing when sampled at $200 \mathrm{~Hz}$. With this setting, there is about $6 \mathrm{~dB}$ reduction of power spectral density at $40 \mathrm{~Hz}$, and $140 \mathrm{~dB}$ reduction of power spectral density at $100 \mathrm{~Hz}$ (EPSON, 2018).

The data logger used was a National Instruments NI CompactRIO-9063. This device consists of a Xilinx Zynq 7000 processor incorporating both an ARM-based microprocessor and a programmable FPGA connected to a four-slot chassis. The chassis can take a variety of different plug-in modules allowing for connection to different types of sensor. Three National Instruments NI 9871 RS-485/RS-422 modules were used. Each of these provided 4 individually controllable sockets, allowing up to 12 devices to be connected in total in the RS-422 one-to-one configuration (NI,2016).

The datalogger runs a custom Real-Time Linux distribution on the ARM portion of the processor. This permits a dedicated LabView-based data logging program to be run at high priority while also using other standard tools such as SSH and Rsync running at a lower priority to transfer data from the datalogger to a server located in Cambridge. The actual data collection is performed by another LabView-based program running on the FPGA portion of the processor. This sends a 'ATtention REQuest DATa' (ATREQDAT) command to all sensors concurrently at a rate of $200 \mathrm{~Hz}$ and then awaits a reply from each sensor. These replies are received in parallel and then marshalled sequentially into a real-time First-In, First-Out (FIFO) buffer which is read by the real-time Linux LabView program and stored on a memory stick. Care had to be taken to ensure that there was no contention for FPGA resources between the different concurrent operations running on the FPGA. For example, each sensor's data acquisition thread had a short memory buffer containing its own copy of the 'ATREQDAT' command.

Communications to and from the datalogger were managed by a Raspberry Pi, which was connected to the data logger via a USB cable. The Raspberry Pi maintained a WiFi connection to a mobile broadband router, and the Raspberry Pi allowed the CompactRIO datalogger to connect through it to the Internet via IP forwarding and masquerading using Linux IP tables.

\section{Figure 6 Data acquisition system}

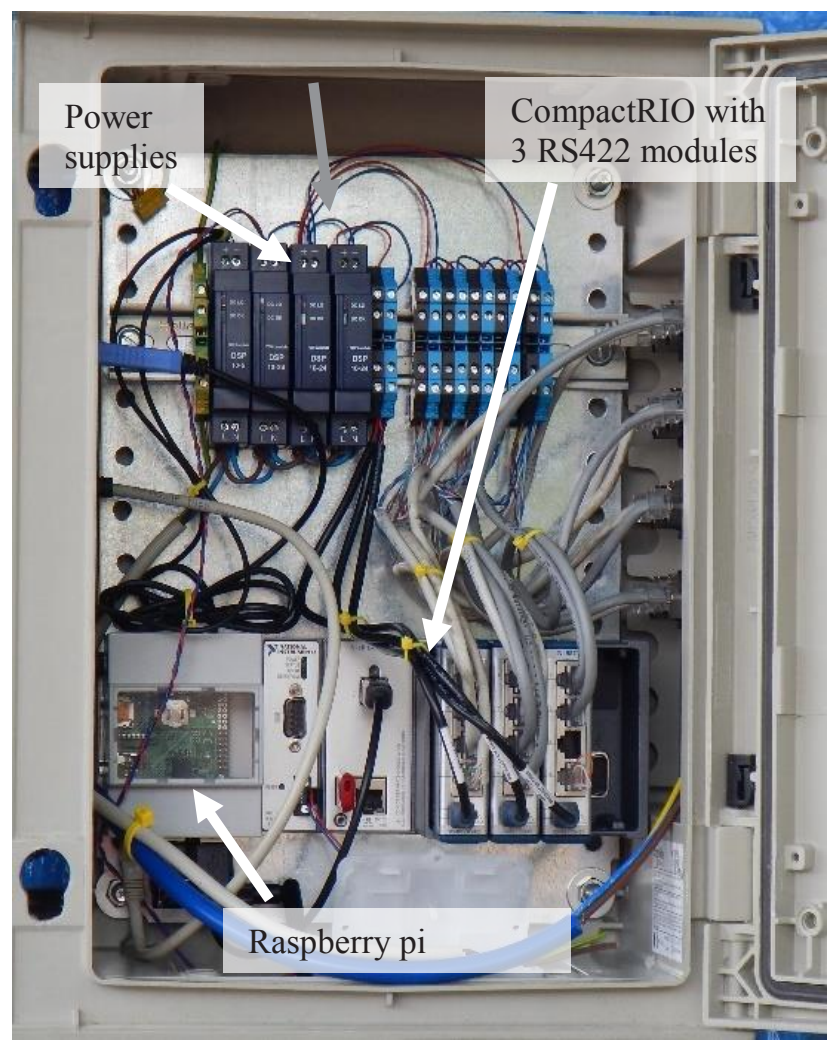


The data acquisition system, comprising data logger, Raspberry Pi and power supplies, is shown in Figure 6. Each of the Epson sensors is connected to the system using external grade CAT 5e cables. Two twisted pairs of the CAT 5e are used for the RS-422 data (one pair for receive, one for transmit) while the other two twisted pairs are used to supply power at $24 \mathrm{~V}$.

In order to capture scour sensitive longitudinal modes of vibration, the sensor arrangement was chosen as shown in Figure 7. Seven M-A550 accelerometers (Nos. 1, 2, 3, 6, 7, 8 and 9) were placed in one vertical plane at the upstream edge of the bridge. Three M-A550 accelerometers (Nos. 4, 5 and 10) were placed halfway along the pier for validation, redundancy and to capture additional modes of vibration. Sensors 3 and 4 did not work possibly due to damage to the sensor cables. All the other 8 sensors provided continuous results thought the project.

A sampling rate of $200 \mathrm{~Hz}$ and a recording period of ten minutes was used for each dataset to get acceptable frequency resolution and enable sufficient averaging when the spectral density is calculated.

Figure 7 Sensor positions on the bridge (Sensor numbers are labelled next to the sensors)

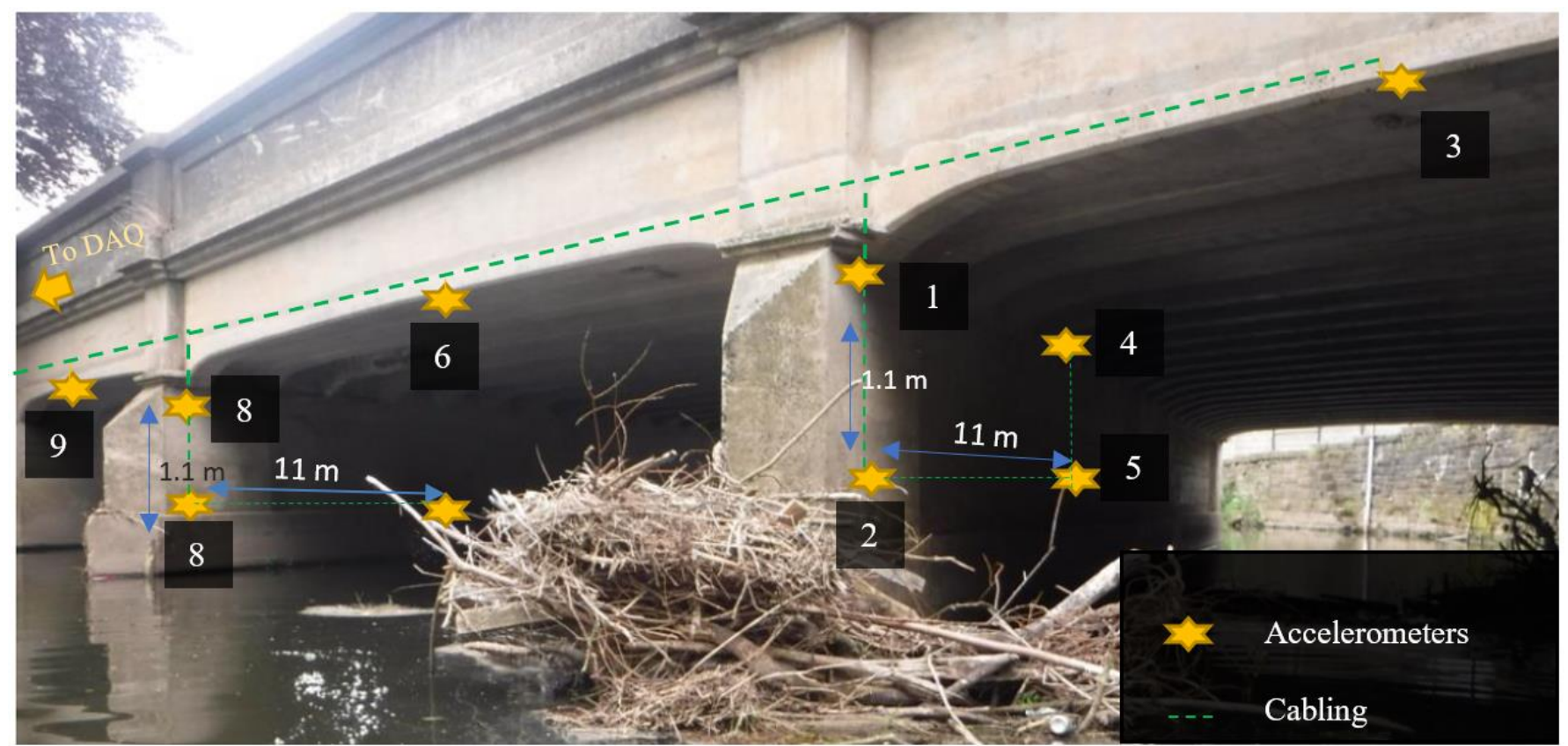

\section{Modal analysis}

All datasets were first pre-processed as follows: (1) uncompress the data files; (2) categorise data into each sensor and remove any datasets with errors in time synchronisation (the error limit for time synchronisation was taken as $250 \mu \mathrm{s}$ ); (3) convert accelerations to $\mathrm{m} / \mathrm{s}^{2}$ and remove the offset of each channel.

Frequency domain decomposition (FDD) was used to capture the frequencies and mode shapes of the bridge from the datasets. In contrast to conventional spectral density functions, FDD combines all sensor channels to show dynamic properties in one function - the singular value of spectral density (Brincker et al., 2017).

FDD was carried out in MATLAB 2018. According to Brincker et al., 2007, the first singular value of the spectral density matrix shows an overlay of the auto spectral density function of modal coordinates. Therefore, a peak in singular values indicates a mode of vibration and the corresponding first singular vector is its mode shape.

The transverse direction of the bridge has lower vibration and lower signal-to-noise ratio than the other directions. Therefore, only the longitudinal and vertical direction sensor channels were taken for FDD analysis. The accuracy of the identified mode shapes was assessed with modal assurance criterion (MAC) (Pastor et al., 2012).

One main assumption made in FDD is that the source of vibration is a wideband excitation. The main source of vibration of Baildon Bridge is traffic, which does not provide a perfect wideband excitation. However, during peak hours, the large number of vehicles over the bridge can be assumed to provide a reasonably wideband excitation. Therefore, datasets at peak traffic hours of 16:00-17:00 on $24^{\text {th }}$ July (before repair works started) were chosen to identify the bridge modes. During this time, there was no change of water level according to a Saltaire water level gauge $1 \mathrm{~km}$ upstream (Environmental Agency UK, 2018). The air temperature measurements of 
sensors shaded under the bridge did not show any change of temperature. Therefore, it could be assumed that there were no environmental changes during this time period. Thus, all datasets in this period should indicate the same bridge modes.

\section{Initial findings}

The singular value plots of the six adjacent datasets are shown in Figure 8. The peak frequencies at $4.5 \pm 0.6 \mathrm{~Hz}$ and $18.75 \pm 0.8$ $\mathrm{Hz}$ were found to be consistent across all datasets. In addition, the mode shapes found at these frequencies showed high modal assurance (over 98\%) when compared with different datasets, as shown in Figure 9.

Figure 10 shows all singular values for one dataset. The first singular value shows five peaks. The range of frequencies that have mode shapes with more than $90 \%$ modal assurance criteria to the modes at the adjacent peaks are shaded. The peaks at $0.58 \mathrm{~Hz}, 10.95 \mathrm{~Hz}$ and $11.95 \mathrm{~Hz}$ were found to be harmonics since the same peak is present in higher singular values (Jacobsen et al., 2017).

Figure 8 First singular value of adjacent datasets showing consistent peaks at $4.5 \mathrm{~Hz}$ and $18.75 \mathrm{~Hz}$

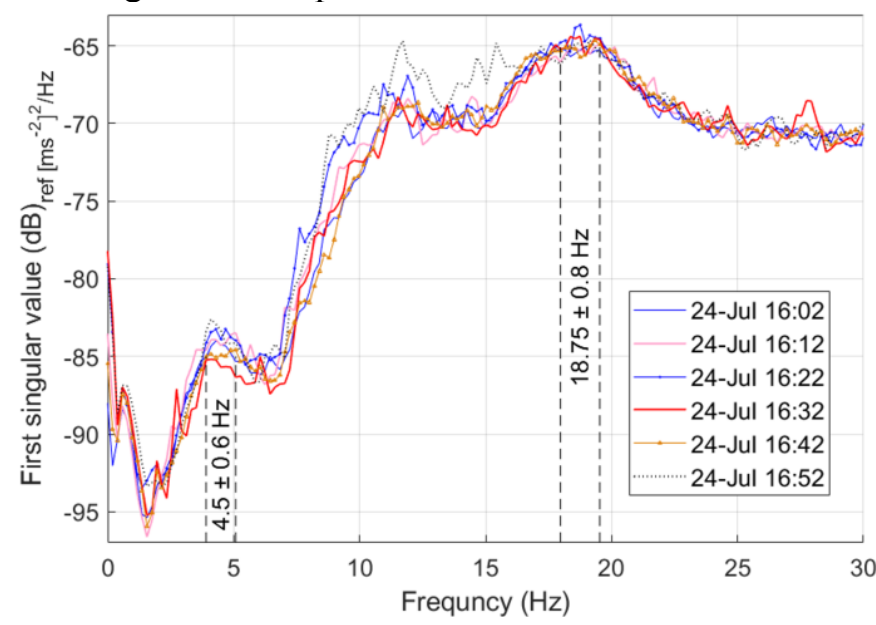

Figure 9 Modal assurance criteria for the dataset combinations at $18.75 \mathrm{~Hz}$ and $4.5 \mathrm{~Hz}$ showing high consistency (above $98 \%$ modal assurance) of mode shapes at both peaks
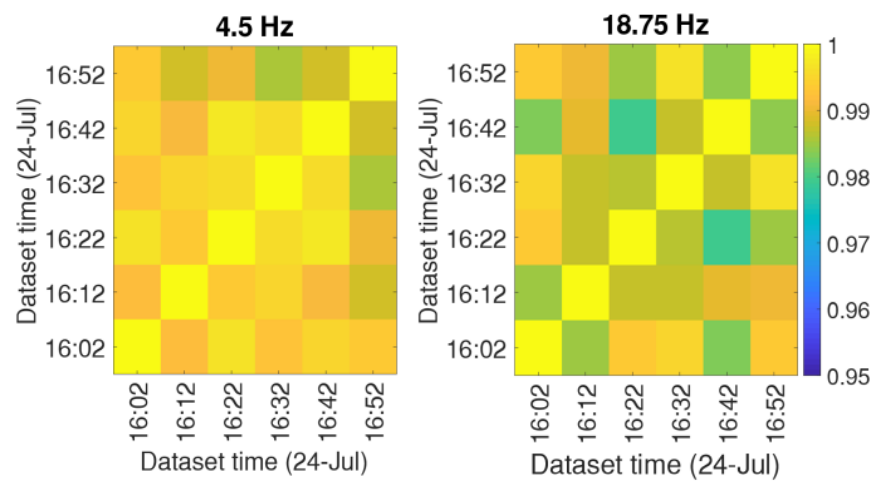

The low-frequency predominantly vertical modes of vibration seen in the initial FE model were not detected in the real bridge. This suggests the vertical skin friction and end bearing at the soil-pile interface is higher than what was assumed with the API design code and $50 \%$ relative density.

Figure 10 Singular values of the dataset at time 16:22. The $90 \%$ MAC region to the mode shape at each first singular value peak is shaded.

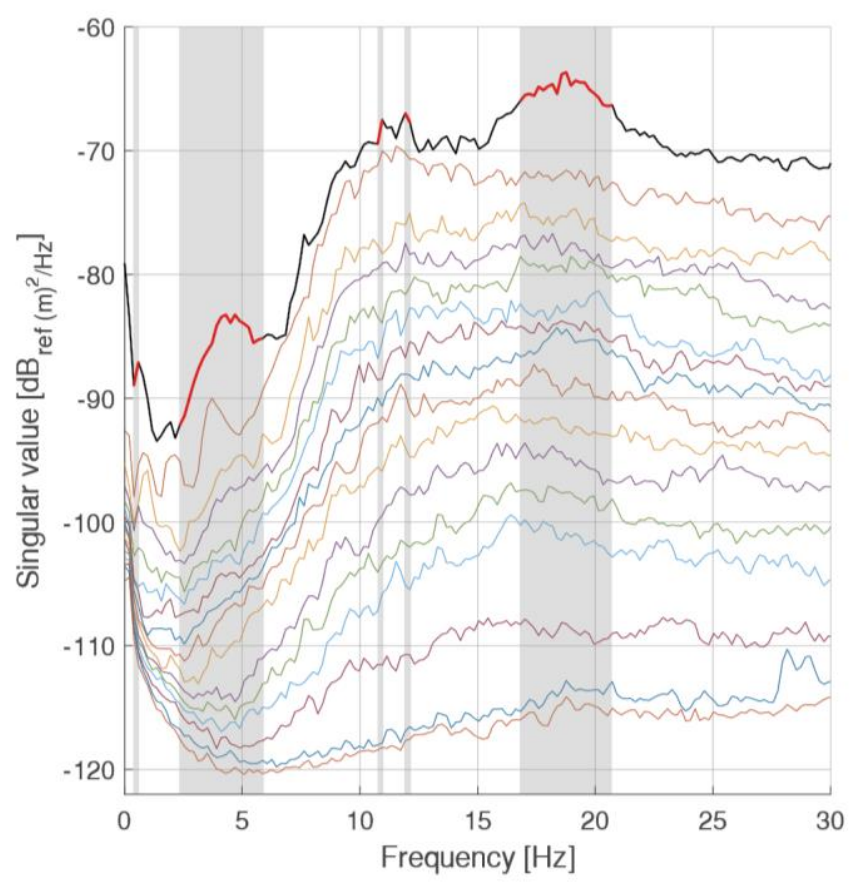

As shown in Table 2, the observed mode at $4.5 \mathrm{~Hz}$ is a sway mode which was not detected with the initial FE model. Since the bridge deck beams are joined by a solid diaphragm wall that had been cast on top of the bridge abutments, a pinned connection was assumed at this interface in the initial FE model. The presence of a sway mode in the real bridge indicates that the bridge abutment-to-beam connections do not provide significant restraint against longitudinal translation. Thus, the FE model was changed (update 1 in Table 2) by releasing the longitudinal translation (i.e. assuming a roller support condition) at each of these interfaces, after which, the FE analysis showed a sway mode matching ( $95 \% \mathrm{MAC})$ to the experimentally observed sway mode.

Table 2 also shows that the observed mode at $18.75 \mathrm{~Hz}$ is a bending mode of the deck (two piers out of phase). The pier vibration matches the initial FE model results (mode detected at $16.37 \mathrm{~Hz}$ ). However, the bridge deck mode shape is different in both the experimentally observed results and the initial FE model. The initial FE model was changed (update 2 in Table 2) by fixing the vertical translation at the base of all piles since no predominantly vertical vibration modes were observed. This change increased MAC of the FE model to $57.8 \%$ from $0.3 \%$. The FE model was then again changed (update 3 ) by releasing the moment capacity at the top of the bridge pier as there is no reinforcement going from bridge beams to bridge pier. This change weakened the modal assurance. 
Table 2: Experimentally observed mode shapes vs mode shapes shown in initial and updated FE model.

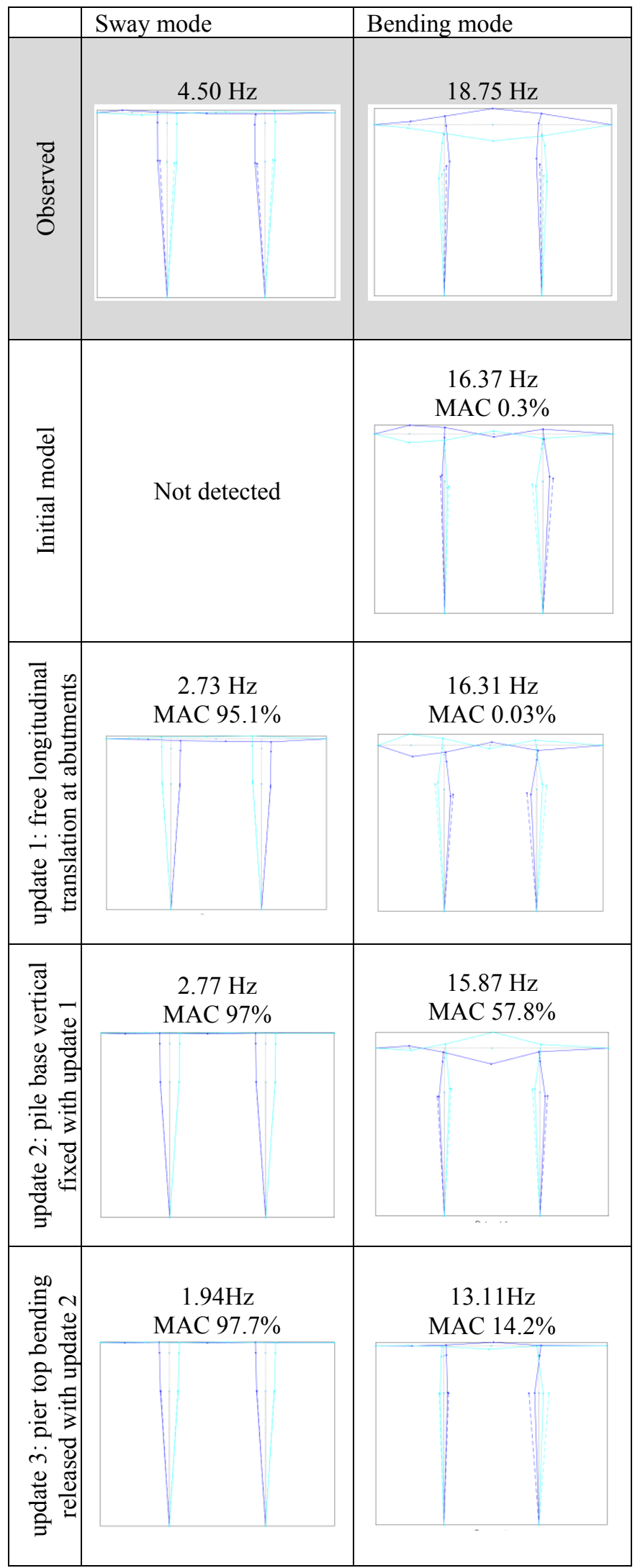

(Mode shapes: modal amplitudes representing the sensors at the upstream edge are marked in solid lines and the sensors at halfway along the piers are marked in dashed lines)
Therefore, the update 2 was adopted in the final model (fixed the vertical translation at the base of bridge piles and released the longitudinal translation at the bridge abutment-beam interface). Further modal assurance may be attainable by increasing the horizontal spring stiffness provided by soil and by assuming partial fixity at abutment-beam interface and at soil-pile base interface.

Scour simulation was carried out as explained in section 4.2 with the final FE model. Table 3 shows the analysis results for all vibration modes up to $20 \mathrm{~Hz}$. The first 2 modes are the most scour sensitive modes of vibration. There is a frequency shift of only $0.14 \mathrm{~Hz}$ for sway mode and $0.54 \mathrm{~Hz}$ for bending mode.

Table 3: Scour simulation results with the updated FE model

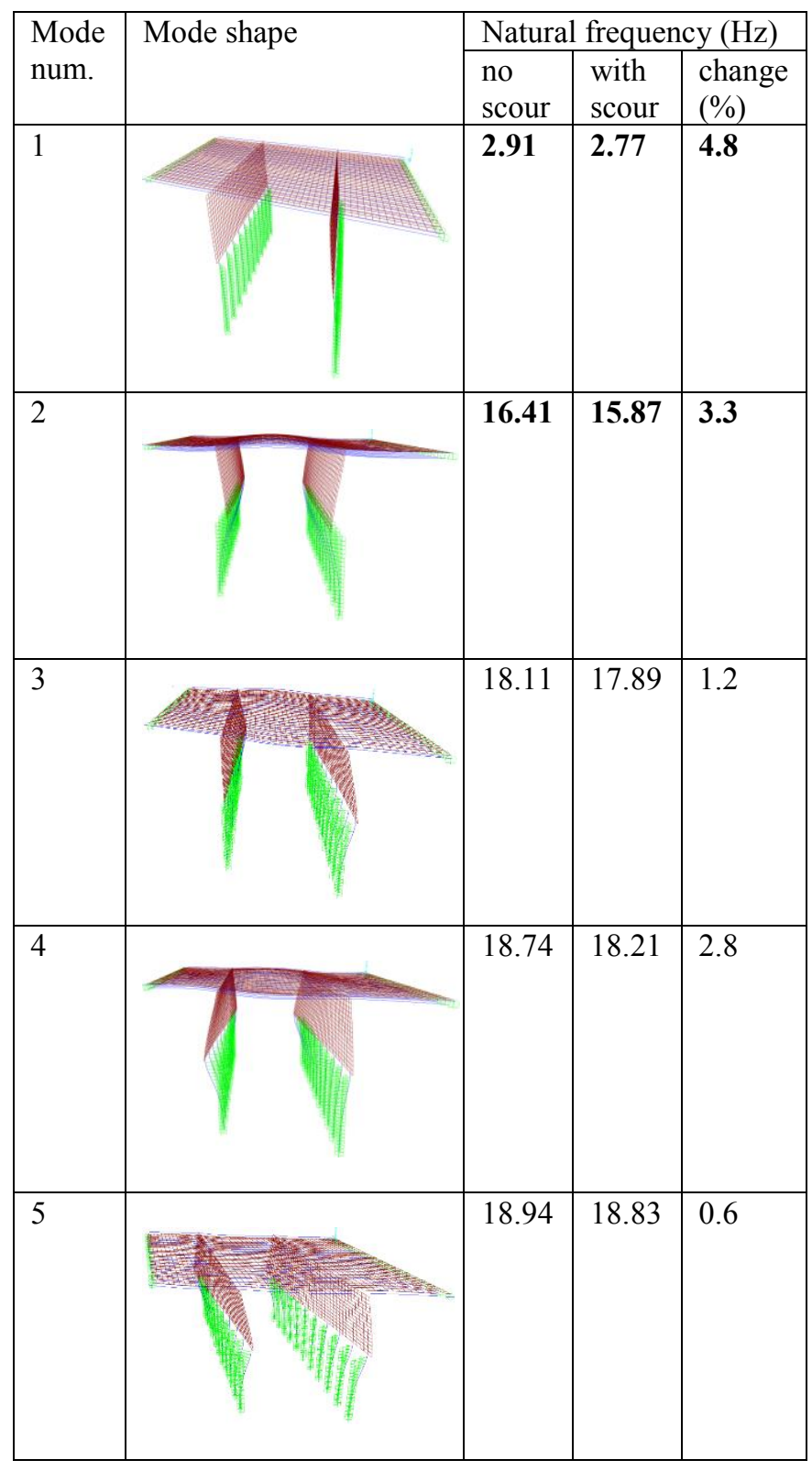




\section{Concluding remarks}

A monitoring system was developed to capture the dynamic properties of Baildon Bridge which had a $1.8 \mathrm{~m}$ deep localised scour hole next to one of its piers. Scour simulation with a $3 \mathrm{D}$ finite-element model found the first longitudinal, pier-bending mode would experience a $0.73 \mathrm{~Hz}$ change due to scour. This mode was observed in the operational modal analysis results with low modal assurance. Experimental results also found a new mode of vibration; thus, the boundary conditions of the bridge were updated. The updated finite-element models found that the natural frequencies of these two modes would change by $0.14-0.54 \mathrm{~Hz}$ due to refilling. The peak picking method with frequency domain decomposition of 6 adjacent datasets found the frequency estimation has a typical error of about 0.6 $0.8 \mathrm{~Hz}$ for this bridge. This inability to obtain high enough frequency resolution indicates a potential challenge for monitoring scour of small-span bridges with vibration-based methods. In the future, other analysis techniques, such as stochastic subspace analysis, will be adopted to improve the accuracy of the modal analysis. In addition, the effect of water level and temperature will be added to finally detect the change of dynamic properties of the bridge due to scour refilling.

\section{Acknowledgements}

We are grateful to the Cambridge Centre for Smart Infrastructure and Construction (Innovate UK grant reference number 920035 and EPSRC grant no EP/N021614/1), for the funding provided for this sensor installation. The $\mathrm{PhD}$ funding provided by the Gates Cambridge Trust is also gratefully appreciated. The research facilities provided by the Laing O'Rourke Centre for Construction Engineering and Technology is gratefully acknowledged. We thank our project partners, Bradford Metropolitan Council and TMS Maritime Ltd, for their assistance and cooperation. Special thanks to Naveed Anwar, Senior Engineer Bradford Council and the TMS scour repair team, Mikael Nickson, Olly Westaway, Rob Martin, Jonathan Peers, Mark and Dave Bridger. We also thank Jenny Roberts of Gaist solutions, and Cumbria Council for the sonar scanning carried out with the BridgeCat vehicle. Final thanks to Peter Knott, Jason Shardelow, Cedric Kechavarzi, Nicky Battista, Haris Alexakis and Tianlei Wu from the Cambridge Centre for Smart Infrastructure and Construction, for their valuable assistance at different stages of this project. Data supporting this paper is available from the University of Cambridge Open Data repository:

https://doi.org/10.17863/CAM.38041

\section{References}

API (2002) Recommended Practice for Planning, Designing and Constructing Fixed Offshore Platforms - Working Stress Design. American Petroleum Institute: DC, United States.

Brincker R et al. (2007) Automated Frequency Domain Decomposition for Operational Modal Analysis. IMAC- $X X V$ : Conference \& Exposition on Structural Dynamics. Society for Experimental Mechanics: FL, USA.
Brownjohn J et al. (2017) Optimised ambient vibration testing of long span bridges. Procedia Engineering. Elsevier B.V. 199: 38-47, doi: 10.1016/j.proeng.2017.09.147.

Chang KC and Kim CW (2016) Modal-parameter identification and vibration-based damage detection of a damaged steel truss bridge. Engineering Structures. Elsevier Ltd 122: 156-173, doi: 10.1016/j.engstruct.2016.04.057.

CIRIA (2015) Manual on Scour at Bridges and Other Hydraulic Structures. Department for Transport: London, UK.

CSI (2016) CSI Analysis Reference Manual for SAP2000, ETABS, SAFE and CSiBridge. Computeres \& Structures, Inc.: CA, USA

Dikanski H et al. (2016) Climate change impacts on railway structures : bridge scour. Proceedings of the Institution of Civil Engineers Engineering Sustainability. ICE UK, doi: 10.1680/jensu.15.00021.

Dutta SC and Roy R (2002) A critical review on idealization and modeling for interaction among soil - foundation structure system. Computers \& Structures 80: 1579-1594.

Environmental Agency UK (2018) Real time flood monitoring data: Archives.

http://environment.data.gov.uk/flood-monitoring/archive EPSON (2018) M-A550AR2x Data Sheet (P/N: E91E60814x). Seiko Epson Corporation: Fujimi, Japan.

Hunt BE (2009) Monitoring Scour Critical Bridges, NCHRP Synthesis 396. The National Academies Press: DC, USA, doi: $10.17226 / 22979$.

Jacobsen, N et al. (2007) Eliminating the Influence of Harmonic Components in Operational Modal Analysis. IMAC-XXIV : A Conference \& Exposition on Structural Dynamics. Society for Experimental Mechanics: MO, USA.

Ko YY, L et al. (2010) Scour Evaluation of Bridge Foundations Using Vibration Measurement. 5th International Conference on Scour and Erosion (ICSE-5). ASCE: CA, USA, 884-293.

Masui Y and Suzuki O (2009) Development of a Soundness Evaluation System for Bridge Substructured. JR EAST Technical Review 14: 65-68.

Melville BW and Coleman SE (2000) Bridge scour. Water Resources Publications: CO, USA.

Murphy RR et al. (2011) Robot-assisted bridge inspection. Journal of Intelligent and Robotic Systems: Theory and Applications 64: 77-95, doi: 10.1007/s10846-010-9514-8.

NI (2016) User manual NI cRIO-9063. National Instruments: TX, USA.

Pastor M et al. (2012) Modal Assurance Criterion. Procedia Engineering 48: 543-548, doi: 10.1016/j.proeng.2012.09.551.

Prendergast LJ et al. (2017) Isolating the location of scourinduced stiffness loss in bridges using local modal behaviour. Journal of Civil Structural Health Monitoring. Springer Berlin Heidelberg, doi: 10.1007/s13349-017-0238-3.

Prendergast LJ et al. (2016) Determining the presence of scour around bridge foundations using vehicle-induced 
vibrations. Journal Of Bridge Engineering In Press(10): 1-14, doi: 10.1061/(ASCE)BE.1943-5592.0000931.

Prendergast LJ et al. (2013) An investigation of the changes in the natural frequency of a pile affected by scour. Journal of Sound and Vibration. Elsevier 332: 6685-6702, doi: 10.1016/j.jsv.2013.08.020.

Rehman SKU et al. (2016) Nondestructive test methods for concrete bridges: A review. Construction and Building Materials. Elsevier 107: 58-86, doi:10.1016/j.conbuildmat.2015.12.011.

Roberts J (2017) BridgeCat: Presentation at the 54th UK Bridge Owners Forum.

http://www.bridgeforum.org/bof/meetings/bof54/BOF54 Scour06 - Roberts - Gaist BridgeCat (no video).pdf

Seo J et al. (2016) Summary Review of Structural Health Monitoring Applications for Highway Bridges. Journal of Performance of Constructed Facilities 30(4): 04015072, doi: 10.1061/(ASCE)CF.1943-5509.0000824.

Shinoda M et al. (2008) Nondestructive Evaluation of Railway Bridge Substructures By. Fourth International Conference on Scour and Erosion. Tokyo, Japan, 285-290.

Shirole AM and Holt RC (1991) Planning for a comprehensive bridge safety assurance program. Transportation Research Record No. 1290, Third Bridge Engineering Conference. CO, USA.

Wardhana K and Hadipriono FC (2003) Analysis of recent bridge failures in the United States. Journal of Performance of Constructed Facilities 17(3): 144-150, doi:10.1061/(Asce)0887-3828(2003)17:3(144).

Zhu L et al. (2018) Development of a high-sensitivitywireless accelerometer for structural health monitoring. Sensors (Switzerland) 18(1): 1-16, doi: 10.3390/s18010262. 